\title{
Early Anti-Coagulation after Ischemic Stroke due to Atrial Fibrillation is Safe and Prevents Recurrent Stroke
}

\author{
Christopher Hahn, Michael D. Hill
}

\begin{abstract}
Background: Patients with acute cardio-embolic stroke from atrial fibrillation (AF) are at risk for recurrence with up to $50 \%$ of recurrent stroke occurring within two weeks of the index event. Anti-coagulation with heparinoids within 48 hours of stroke has been shown to increase risk of symptomatic intracranial hemorrhage (ICH) with no clear benefit on early stroke recurrence. Methods: This study was a retrospective chart review of consecutive patients who were admitted to the stroke service at the Foothills Medical Centre between 2009 and 2011. All patients with an acute stroke with a cardio-embolic etiology and a diagnosis of atrial fibrillation were reviewed. We hypothesized that anti-coagulation within two weeks of stroke, appropriately begun because of a diagnosis of AF, decreased rates of recurrent stroke without causing an increase in rates of symptomatic ICH. Results: Between 2009-2011, 324 patients were identified with cardio-embolic stroke secondary to AF. Within two weeks of stroke onset $61.4 \%$ (199/324) of patients were therapeutic on anti-coagulation. Patients who were anti-coagulated had a smaller median index stroke volume (3.2 $\mathrm{ml} \mathrm{vs} 18.4 \mathrm{ml})$. Three ( $0.9 \%)$ patients suffered a clinically significant ICH. Recurrent stroke occurred in 11 patients $(3.4 \%)$ within the two-week period. Therapeutic anticoagulation within two weeks of initial stroke was associated with a decreased risk of recurrent stroke (RR 0.1, 95\% CI 0.03-0.64). Conclusions: Anti-coagulation within two weeks of acute stroke in patients with AF appears to be safe among patients with smaller infarcts and prevents early recurrent infarction.
\end{abstract}

RÉSUMÉ: L'anticoagulation précoce après un accident vasculaire cérébral ischémique dû à la fibrillation auriculaire est sûre et prévient la survenue d'un deuxième accident vasculaire cérébral. Contexte: Les patients atteints d'un accident vasculaire cérébral (AVC) cardioembolique dû à la fibrillation auriculaire (FA) sont à risque de présenter un deuxième AVC qui survient, chez $50 \%$ des cas, au cours des deux semaines suivant le premier AVC. Il a été démontré que l'anticoagulation au moyen d'héparinoïdes dans les 48 heures suivant l'AVC augmente le risque d'hémorragie intracrânienne symptomatique (HICS) sans procurer de bénéfice certain quant à la survenue d'un deuxième AVC. Méthode: Nous avons revu rétrospectivement les dossiers de patients consécutifs qui ont été hospitalisés à l'unité de soins spécialisés en l'AVC au Foothills Medical Centre entre 2009 et 2011 . Tous les dossiers des patients atteints d'un AVC aigu d'étiologie cardioembolique, chez qui un diagnostic de FA avait été posé, ont été revus. Nous avons émis l'hypothèse que l'anticoagulation dans les deux semaines suivant l'AVC, suite à un diagnostic de FA, diminuait le taux de survenue d'un deuxième AVC sans augmenter le taux d'HICS. Résultats : Entre 2009 et 2011, 324 patients atteints d'un AVC cardioembolique secondaire à la FA ont été identifiés. Au cours des deux semaines suivant le début de l'AVC, 61,4\% des patients (199/324) ont reçu une dose thérapeutique d'anticoagulant. Le volume médian de l'AVC initial des patients qui ont été anticoagulés était plus petit $(3,2 \mathrm{ml} v \mathrm{vs} 18,4 \mathrm{ml})$. Trois patients $(0,9 \%)$ ont subi un AVC important au point de vue clinique. Onze patients $(3,4 \%)$ ont subi un deuxième AVC au cours des deux semaines suivant le premier. Une anticoagulation thérapeutique au cours des deux semaines suivant l'AVC initial était associée à une diminution du risque d'un deuxième AVC (RR 0,1; IC à 95\% de 0,03 à 0,64). Conclusions: L'anticoagulation au cours des deux semaines qui suivent un AVC aigu chez les patients atteints de FA semble être sûre chez ceux qui sont atteints d'infarctus cérébraux plus petits et prévient la survenue précoce d'un second infarctus.

Keywords: Anti-coagulation, atrial fibrillation, ischemic stroke

doi:10.1017/cjn.2015.7

Can J Neurol Sci. 2015; 42: 92-95

Atrial fibrillation is the most common arrhythmia in older adults and a common cause of ischemic stroke, representing upwards of $15 \%$ of all strokes. ${ }^{1}$ Anti-coagulation with warfarin and other newer agents effectively reduces stroke risk but also increases rates of both intra and extra cranial hemorrhage. ${ }^{2,3}$ In the last several years, new oral anticoagulant medications have been introduced that are at least as effective if not better than warfarin for stroke prevention. None of these drugs has been assessed for secondary prevention of stroke in the acute stage. Patients with acute stroke due to atrial fibrillation present a management dilemma: when is it safe to start or restart anti-coagulation?
Past trials have not shown benefit to early anticoagulation with fixed dose, subcutaneous, unfractionated or low molecular weight heparin $^{4-8}$ and data on use of novel oral anticoagulants in the

From the Calgary Stroke Program, Department of Clinical Neurosciences, Hotchkiss Brain Institute, University of Calgary, Calgary, AB, Canada.

Received June 30, 2014. FinAl Revisions Submitted October 30, 2014 Correspondence to: Michael D. Hill, Calgary Stroke Program, Department of Clinical Neurosciences, Hotchkiss Brain Institute, University of Calgary, Foothills Hospital, Rm 1242A, $140329^{\text {th }}$ Street NW, Calgary, Alberta, T2N 2T9, Canada.

Email: michael.hill@ucalgary.ca 
immediate post-stroke period is lacking. ${ }^{9}$ Nonetheless, recurrent stroke rates are highest in the 90 days following the initial event with $50 \%$ occurring within two weeks. ${ }^{10}$ Despite treatment with acetylsalicylic acid (ASA), rates of recurrence in patients with atrial fibrillation have been reported as high as $7.5 \%$ within this two week period. ${ }^{5}$

Current guidelines on the timing of anticoagulation in acute stroke due to atrial fibrillation are based upon expert opinion and suggest that patients with smaller infarct volumes and lack of hemorrhagic transformation may be safely anti-coagulated to prevent recurrent stroke without causing intracranial hemorrhage. We sought to assess this hypothesis by evaluating hemorrhage rates and re-infarction rates among patients with atrial fibrillation who were admitted to the stroke service at the Foothills Medical Centre, Calgary, Alberta and treated with anti-coagulants.

\section{MeTHODS}

This study was approved by the University of Calgary Conjoint Health Research Ethics Board. All patients consecutively entered into the Foothills Hospital Stroke Unit Database between January $1^{\text {st }}, 2009$ and December $31^{\text {st }}, 2011$ were reviewed for inclusion in this study.

All patients with a stroke or transient ischemic attack whose mechanism was determined by the attending stroke neurologist as 'cardioembolic' or 'unknown/cryptogenic' were screened for inclusion. All patients in the 'unknown/cryptogenic' category were cross referenced with the cardiac diagnostic database for a cardiologist verified diagnosis of atrial fibrillation on electrocardiogram or Holter monitor within one year of stroke.

Initial patient characteristics were collected from the regional electronic health record and via paper chart review. A diagnosis of stroke was defined as acute onset of persistent (lasting more than 24 hours) neurologic dysfunction with no other non-vascular cause or transient neurologic symptoms with imaging confirmation of new ischemic stroke. Imaging was reviewed and stroke volume was estimated using the ABC/2 method ${ }^{11}$ on computed tomography (CT) or magnetic resonance (MR) diffusion weighted imaging within 72 hours of admission. Patients were excluded if they had no evidence of stroke on MR imaging, had a diagnosis of infective endocarditis, had evidence of greater than $50 \%$ carotid stenosis ipsilateral to their stroke or were transferred out of the province before follow-up was completed.

Primary outcomes were recurrent ischemic stroke and symptomatic intra-cranial hemorrhage within two weeks of initial stroke or death at three months. Recurrent stroke was defined as new neurological symptoms corresponding to a different territory than the initial infarction with no clear hemorrhagic, infectious or metabolic cause, or imaging evidence of new infarction of presumed embolic origin. Symptomatic hemorrhage was defined as a presence of new intracranial blood on imaging with a change in neurological examination corresponding to the area of hemorrhage. An unchanged neurological exam at follow-up or followup imaging study within three months with no new stroke was considered equivalent to absence of the primary outcomes. Follow-up examinations were abstracted from the inpatient chart as documented. Patients who were discharged from hospital before two weeks had follow-up examination obtained from Stroke Prevention Clinic charts at three month follow-up. For patients with no in-hospital or clinic follow up, a search of the
Alberta Health Services administrative database was undertaken looking for admission diagnosis codes representing death, ischemic stroke and intra-cranial hemorrhage. Patients with no repeat admission in the two week period were assumed to have absence of recurrent stroke or symptomatic intracranial hemorrhage.

Therapeutic measures used for treatment of acute stroke and prevention of recurrent stroke and post stroke complications were recorded. This included administration of recombinant tissue plasminogen activator, use of intra-arterial therapy, use of antiplatelet agents, administration of heparinoids for prophylaxis of deep vein thrombosis, choice of anti-coagulation for atrial fibrillation and time to achievement of therapeutic anti-coagulation. A patient was considered therapeutic at the time of the first dose of low molecular weight heparin (LMWH), when the international normalized ratio (INR) was greater than 2.0 for more than two days, when the partial thromboplastin time (PTT) was greater than 55.0 for two consecutive measurements or the time when Dabigatran was started. Data were reported using standard descriptive statistics.

\section{ReSUlTS}

In the three year study period, 624 patients were admitted to the stroke unit with a history or cardiac diagnostic test demonstrating atrial fibrillation and a presumed cardio-embolic or unknown stroke mechanism. After chart review, 324 patients were included for analysis. The remainder were excluded on the basis of lack of ischemic stroke (291) or transfer out of province before the end of the follow-up period (9). One hundred and sixty one patients lacked follow-up in hospital or in the stroke prevention clinic and follow up was obtained from searches on the administrative database.

Two hundred and forty three patients $(75 \%)$ received at least one dose of an anti-coagulant (heparin, warfarin, low molecular weight heparin or Dabigatran) within two weeks of stroke. Of those started on an anticoagulant, follow-up data regarding INR values were available in 241 patients (99\%) with $203(81.9 \%)$ achieving therapeutic drug levels within two weeks. Four of those patients were started on anticoagulation after a recurrent stroke. Among the patients therapeutic within two weeks, $156(77 \%)$ were therapeutic within one week and 77 patients (38\%) within 48 hours. The mean time to therapeutic anti-coagulation was 4.0 days with $50 \%$ therapeutic by three days. Warfarin was the most common anti-coagulant used with 174 (85.7\%) patients receiving at least one dose within the two week period. Unfractionated heparin was given to 77 patients, LMWH to 48 and only 19 received dabigatran. MR imaging was used for stroke volume measurement in 132 patients $(41 \%)$ and $\mathrm{CT}$ in the remainder. Patients who were anti-coagulated had a smaller median stroke volume (3.2 $\mathrm{ml}$ vs $18.4 \mathrm{ml})$, were more commonly therapeutic on anticoagulation at presentation $(10.3 \%$ vs $1.6 \%)$, more likely to have an echocardiogram showing echocardiographic predictors of cardioembolism $^{12,13}(18.9 \%$ vs $7.3 \%)$ and had non-significantly lower median National Institutes of Health stroke scale (NIHSS) at presentation (5 versus 7) (Table).

Eleven patients $(3.4 \%)$ had a recurrent ischemic stroke within two weeks of the initial event. Two of the patients with recurrent ischemic stroke were therapeutic on anti-coagulants at time of stroke. Therapeutic anti-coagulation within two weeks of initial 
Table: Baseline Patient Characteristics

\begin{tabular}{|c|c|c|}
\hline & Not Therapeutic on Anticoagulation $n=125$ & $\begin{array}{l}\text { Therapeutic on Any Anticoagulant Before } 2 \text { weeks or } \\
\text { Before Recurrent Stroke } n=199\end{array}$ \\
\hline Mean Age & $76.2\left(\mathrm{SD}^{*} 10.8\right)$ & $76.4(\mathrm{SD} 10.8)$ \\
\hline Female Sex & $59(47.2 \%)$ & $109(54.8 \%)$ \\
\hline Hypertension & $95(76.0 \%)$ & $139(69.9 \%)$ \\
\hline Diabetes Mellitus & $27(21.6 \%)$ & $34(17.1 \%)$ \\
\hline Coronary Artery Disease & $37(29.6 \%)$ & $46(23.1 \%)$ \\
\hline Previous Stroke/TIA & $32(25.6 \%)$ & $49(24.6 \%)$ \\
\hline Peripheral Vascular Disease & $3(2.4 \%)$ & $4(2.0 \%)$ \\
\hline Dyslipidemia & $25(20.0 \%)$ & $46(23.1 \%)$ \\
\hline Valvular Heart Disease & $11(8.8 \%)$ & $13(6.5 \%)$ \\
\hline Current Tobacco Use & $15(12 \%)$ & $23(11.6 \%)$ \\
\hline Therapeutic on Anticoagulation before Initial Stroke & $2(1.6 \%)$ & $21(10.6 \%)$ \\
\hline ASA use before Stroke & $53(43.2 \%)$ & $81(40.7 \%)$ \\
\hline EtOH Use (>2 drinks/day) & $6(4.8 \%)$ & $8(4.0 \%)$ \\
\hline Congestive Heart Failure & $16(12.8 \%)$ & $28(14.1 \%)$ \\
\hline Median Stroke Size by ABC/2 (ml) (range) & $18.4(0-354)$ & $3.2(0-402)$ \\
\hline Microbleeds on MRI & $3(6.5 \%)$ & $5(5.8 \%)$ \\
\hline Platelet count $\left(\times 10^{9}\right.$ cells $\left./ \mathrm{L}\right)$ & 218 & 221 \\
\hline Glucose $(\mathrm{mM})$ & 7.1 & 6.9 \\
\hline Median Initial NIHSS (range) & $7(0-28)$ & $5(0-32)$ \\
\hline Initial Blood Pressure $(\mathrm{mm} \mathrm{Hg})$ & $151 / 82$ & $151 / 83$ \\
\hline Abnormal Echocardiogram $^{\dagger}$ & $5(6.9 \%)$ & $21(19.6 \%)$ \\
\hline
\end{tabular}

${ }^{*} \mathrm{SD}=$ Standard deviation; TIA = transient ischemic attack; ASA = acetylsalicylic acid; EtOH = ethanol; $\mathrm{ICH}=$ intracerebral hemorrhage; $\mathrm{MRI}=$ magnetic resonance imaging; INR = international normalized ratio; PTT = partial thromboplastin time; NIHSS = National Institutes of Health Stroke Scale score. ${ }^{\dagger}$ Echocardiogram showing atrial thrombus, low left ventricular ejection fraction, atrial septal aneurysm, grade IV aortic atheroma, low peak atrial appendage flow or dense spontaneous echo contrast.

stroke was associated with decreased risk of recurrent stroke (RR $0.14,95 \%$ CI 0.03-0.64). This remained significant after multivariable analysis adjusting for age and sex. Three patients $(0.9 \%)$ had a clinically significant intracerebral hemorrhage (ICH); one patient was actively anti-coagulated at the time of $\mathrm{ICH}$, one was taking ASA only and one was on ASA and low dose enoxaparin for prevention of deep vein thrombosis. The anti-coagulated patient became therapeutic 11 days after the index stroke, had an initial stroke volume of $189 \mathrm{ml}$ and the ICH occurred on day 14 . Use of anti-coagulation was not significantly associated with symptomatic intracranial hemorrhage. Univariate predictors of ICH included higher NIHSS score (RR 1.2, 95\% CI 1.09-1.3, per point increase in NIHSS) with younger age being protective against ICH (RR 0.9, 95\% CI 0.87-0.97, per year).

Twenty-two patients $(7 \%)$ were deceased at three months. In univariate analysis, use of anticoagulation was associated with a reduced risk of death at three months irrespective whether or not a therapeutic INR was achieved within two weeks (RR $0.1, p<0.01$ 95\% CI 0.04-0.26). Other predictors of death included large stroke size, anterior cerebral artery strokes, use of aspirin alone, use of low dose heparin for deep vein thrombosis prophylaxis, high systolic blood pressure in hospital and higher NIHSS scores. After multivariable analysis, use of anticoagulation (OR 0.15, $\mathrm{p}<0.01$ 95\% CI 0.04-0.6) was protective against death and use of deep vein thrombosis prophylaxis (OR 6.0, $\mathrm{p}<0.0195 \%$ CI 1.8-19.9) was associated with death.

\section{Discussion}

We suggest that early anti-coagulation among patients with smaller infarct sizes prevents recurrent stroke without significantly increasing the risk of intracranial hemorrhage. Only one patient who was anti-coagulated suffered an ICH despite $38 \%$ of anticoagulated patients becoming therapeutic within 48 hours of stroke symptom onset. This result lies in contrast to previous studies suggesting increased rates of $\mathrm{ICH}^{4,5,7,14}$ with early anticoagulation. We believe that this observation is directly related to the difference in baseline infarct size between the two groups. Our population had less severe and smaller volume strokes, reflecting current clinical practice. A previous study looking specifically at use of heparin acutely in cardio-embolic stroke found that risk of hemorrhage was associated with higher mean PTT ratios but not stroke severity. ${ }^{15} \mathrm{We}$ did not find this association but this may be a reflection of the relative infrequency of $\mathrm{ICH}$ in our group. Additionally because of the rarity of ICH the study was underpowered to establish a threshold infarct size above which the risk of ICH increases. Our calculation of infarct size is also limited by the use of both CT and MR imaging. 
Magnetic resonance imaging was used as the preferred measurement medium whenever possible but only available in 132 patients.

The decrease in recurrent stroke is in keeping with a sub-group analysis of International Stroke Trial (IST) showing significantly less recurrent stroke in patients allocated to heparin versus no heparin $^{6}$ and meta-analysis of anticoagulation in acute stroke which showed a trend for benefit in recurrent stroke. ${ }^{16}$ In contrast, Heparin in Acute Embolic Stroke Trial

(HAEST) showed no significant difference in stroke rates between patients given dalteparin compared to ASA alone. However HAEST excluded patients who clinicians felt had a clear indication for anticoagulant therapy or a clear contraindication for antithrombic therapy' and who were on oral anticoagulation at time of stroke. ${ }^{5}$ Given that $24 \%$ of patients in our study were orally anticoagulated, the difference in results may be explained by the difference in baseline patient populations. Furthermore, our population of anti-coagulated patients had a significantly higher incidence of echocardiographic predictors of embolic stroke. This element of selection bias may also contribute to the benefit of anti-coagulation seen in our data.

Use of anticoagulation was also protective against death. This may be due to confounding by indication, rather than a true medical effect. Patients with larger strokes, who are most at risk of death due to stroke severity, tended not to be anti-coagulated acutely. The positive association with use of deep vein thrombosis prophylaxis and death may be similarly explained. Patients with more disabling strokes and worse mobility were likely to be started on deep vein thrombosis prophylaxis.

This study is limited by its small sample size and retrospective nature. There is potential for selection bias in our follow up procedures: 161 patients had no follow-up in hospital or in the stroke clinic and were assumed to be free of recurrent stroke or hemorrhage if they had no admission found in the administrative database. Admissions to community hospitals may have been missed if they were not correctly coded as a stroke or hemorrhage. We were also unable to address the impact of recurrent stroke on longterm functional outcome. Nonetheless, it provides evidence of safety and preliminary evidence of the effectiveness of early anticoagulation for acute stroke patients.

There is a need for large randomized trials to examine the early use of anticoagulants, particularly the novel oral anticoagulants because of their ease of use and potentially better stroke prevention efficacy. Using echocardiographic parameters to select patients at higher risk of recurrent embolism may be helpful in such a study. Safe in-hospital initiation of anticoagulation is likely to result in better long-term compliance with anticoagulant therapy and reduced stroke occurrence among patients with atrial fibrillation.

\section{Statement of Authorship}

$\mathrm{CH}$ conducted the chart reviews, primary analysis and wrote the first draft. MDH provided overall supervision, review of analysis and revision of the paper.

\section{ACKNowledgements AND Funding}

$\mathrm{MDH}$ is funded by Alberta Innovates Health Solutions and by the Heart \& Stroke Foundation of Alberta-Hotchkiss Brain Institute Professorship in Stroke Research.

\section{Disclosures}

Authors have nothing to disclose.

\section{REFERENCES}

1. Wolf PA, Abbott RD, Kannel WB. Atrial fibrillation as an independent risk factor for stroke: the Framingham Study. Stroke. 1991;22:983-8.

2. Hart RG, Pearce LA, Aguilar MI. Meta-analysis: antithrombotic therapy to prevent stroke in patients who have nonvalvular atrial fibrillation. Ann Intern Med. 2007;146:857-67.

3. Connolly SJ, Ezekowitz MD, Yusaf S, et al. Dabigatran versus warfarin in patients with atrial fibrillation. $\mathrm{N}$ Engl $\mathrm{J}$ Med. 2009;361:1139-51.

4. The International Stroke Trial (IST): a randomised trial of aspirin, subcutaneous heparin, both, or neither among 19435 patients with acute ischaemic stroke. International Stroke Trial Collaborative Group. Lancet. 1997;349:1569-81.

5. Berge E, Abdelnoor M, Nakstad PH, et al. Low molecular-weight heparin versus aspirin in patients with acute ischaemic stroke and atrial fibrillation: a double-blind randomised study. HAEST Study Group. Heparin in Acute Embolic Stroke Trial. Lancet. 2000;355:1205-10.

6. Saxena R, Lewis S, Berge E, et al. Risk of early death and recurrent stroke and effect of heparin in 3169 patients with acute ischemic stroke and atrial fibrillation in the International Stroke Trial. Stroke. 2001;32:2333-7.

7. Dahl T, Friis P, Abildgaard U. Dalteparin in Acute Ischemic Cerebrovascular Disease: A Safety Study. Cerebrovasc Dis. 1997;7: 28-33.

8. Kay R, Wong KS, Woo J. Pilot study of low-molecular-weight heparin in the treatment of acute ischemic stroke. Stroke. 1994;25:684-5.

9. Ganetsky M, Babu KM, Salhanick SD, et al. Dabigatran: review of pharmacology and management of bleeding complications of this novel oral anticoagulant. J Med Toxicol. 2011;7:281-7.

10. Johnston SC, Gress DR, Browner WS, et al. Short-term prognosis after emergency department diagnosis of TIA. JAMA. 2000; 284:2901-6.

11. Pedraza S, Puig J, Blasco G, et al. Reliability of the $A B C / 2$ method in determining acute infarct volume. J Neuroimaging. 2012;22:155-9.

12. Atrial Fibrillation Investigators. Echocardiographic predictors of stroke in patients with atrial fibrillation: a prospective study of 1066 patients from 3 clinical trials. Arch Intern Med. 1998;158:1316-20.

13. Zabalgoitia M, Halperin JL, Pearce LA, et al. Transesophageal echocardiographic correlates of clinical risk of thromboembolism in nonvalvular atrial fibrillation. Stroke Prevention in Atrial Fibrillation III Investigators. J Am Coll Cardiol. 1998;31:1622-6.

14. Low molecular weight heparinoid, ORG 10172 (danaparoid), and outcome after acute ischemic stroke: a randomized controlled trial. The Publications Committee for the Trial of ORG 10172 in Acute Stroke Treatment (TOAST) Investigators. JAMA. 1998;279:1265-72.

15. Chamorro A, Vila N, Ascasco C, Blanc R. Heparin in acute stroke with atrial fibrillation: clinical relevance of very early treatment. Arch Neurol. 1999;56:1098-102.

16. Paciaroni M, Agnelli G, Micheli S, Caso V, et al. Efficacy and safety of anticoagulant treatment in acute cardioembolic stroke: a metaanalysis of randomized controlled trials. Stroke. 2007;38:423-30. 